\title{
Medical Journal Impact Factor 2013: ongoing task of getting the right balance
}

\author{
P. Riss • P. L. Dwyer
}

Published online: 30 September 2014

(C) The International Urogynecological Association 2014

At the end of July 2014, the Medical Journal Impact Factor for 2013 was released. This event is always eagerly awaited as if the fate of the scientific publishing business as a whole and of individual editors and authors hangs in the balance. There is no doubt that the impact factor is of major importance and - as the name implies - impact. It is a yardstick against which the success of journals is measured and a metric of importance for the careers of many researchers and clinicians. However, this is not the end of the story. Other factors of importance to authors in deciding on a journal in which to publish is who will be reading my research, and what are the chances of my research being published and how quickly.

The new Impact Factor (IF) 2013 for the International Urogynecology Journal (IUJ) is 2.161 . This is virtually unchanged from the previous year, when the IF was 2.169. The IUJ is now ranked 27 of 78 in the category Obstetrics and Gynecology and 26 of 75 in the category Urology and Nephrology, also practically unchanged from 2012 [1].

When discussing the impact factor, it is always good to remember how it is calculated and, as a consequence, which factors influence it. The calculation is citations in 2013 of articles published in 2012 and 2011, divided by number of articles published in 2012 and $2011(1005 / 465=2.161)$. Generally speaking, the impact factor is a balance between how often articles are cited and the total number of articles published in the journal. It is also important to remember that it embodies very specific time frames, representing the relationship between the number of cites (in 1 year) and the

P. Riss $(\square)$

Department of Obstetrics and Gynecology, Medical University

Vienna, Waehringer Guertel 18-20, 1090 Vienna, Austria

e-mail: priuj11@gmail.com

P. L. Dwyer

Department of Urogynaecology, Mercy Hospital for Women and

Melbourne University, Melbourne, Australia number of articles published in the preceding 2-year period. More citations mean a higher impact factor, but only in relationship to the number of articles published. Articles that may be of interest to readers but are rarely cited in other articles detract from the impact factor.

At the IUJ, we are proud to publish many articles. In 2013, we published 286 articles on 2,123 pages. The IUJ is six of 78 in Obstetrics and Gynecology and seven of 75 in Urology and Nephrology in number of articles published. We have purposely increased the number of articles published over recent years without lowering our acceptance rate of approximately $50 \%$ or our high standard of publication. We believe that this offers the best opportunities for publishing for our international authors and makes for interesting content for our readers. If we were able to refuse manuscripts that we think would not be cited, therefore reducing the number of articles we publish each year, the impact factor of the IUJ would increase. What we aim for, however, is a balance between publishing highly cited work and providing a journal covering the entire range of topics in the subspecialty of urogynecology.

There are metrics that show we may be doing the appropriate thing. Apart from the impact factor, the total number of citations - not limited to the year of article publication - is a metric that indicates a journal's reach and influence. In 2013, articles from the IUJ were cited 3,977 times in other scientific articles. This would rank the IUJ 19 of 78 in Obstetrics and Gynecology and 15 of 75 in Urology and Nephrology, higher than the ranking according to the impact factor (27 and 26, respectively). We believe that these numbers show the overall reach of the IUJ and how useful and relevant its articles are to authors in general.

Recently, the h5-index has been gaining attention. It was originally created to measure the impact of publications of an individual researcher, but Google Scholar has been applying it to journals [2]. The h5-index is for articles published in the prior 5 complete years. By definition, it is the largest number 
$\mathrm{h}$, such that $\mathrm{h}$ articles published in 2009-2013 have at least $\mathrm{h}$ citations each [3]. As of June 2014, the h5-index for the IUJ was 35, giving it a rank number of 15 in Obstetrics and Gynecology and 20 in Urology and Nephrology. The advantage of the h5-index is that it is not influenced by outliers, i.e., very highly cited papers. It is not released by a publishing company once a year but freely available on Google Scholar where it is regularly updated $[4,5]$.

However, it is best not to overestimate the importance of any one metric. Surveys by the International Urogynecology Association (IUGA) show that what members value most are the IUJ and the annual meetings of the society. Readers expect a journal to be of interest and relevant for their work. They want their journal to have a high reputation, and this certainly includes a high impact factor. As editors of the IUJ, we continue to pay close attention to relevant metrics and the international reputation of our journal. In this, we depend on your continuing loyalty and support.

\section{References}

1. Riss P, Dwyer PL (2013) The 2012 IUJ impact factor: another step forward? Int Urogynecol J 24:1591-1592. doi:10.1007/ s00192-013-2197-5

2. $\mathrm{H}$ factor. http://en.wikipedia.org/wiki/H-index. Accessed 17 September 2014

3. Google Scholar Metrics. http://scholar.google.com/intl/en/scholar/ metrics.html. Accessed 17 September 2014

4. h5-index Top publications - Gynecology \& Obstetrics. http:// scholar.google.com/citations?view_op=top_venues\&hl=en\&vq= med_gynecologyobstetrics. Accessed 17 September 2014

5. h5-index Top publications - Urology \& Nephrology. http://scholar. google.com/citations?view_op=top_venues\&hl=en\&vq=med urologynephrology. Accessed 17 September 2014 